\title{
Editorial
}

\section{Surviving Sepsis Campaign 2021: a summary of the new recommendations}

Sepsis is the result of the hosts' response to an infectious agent, leading to organ dysfunction (1). It is a very common syndrome, estimated to have occurred in 48.9 million people in 2017, which represented an incidence of 677.5 (535.7-876.1) cases per 100,000 in the same year (2). It represents the cause of 1 in every 5 deaths annually worldwide (3), affecting especially small children, the elderly and people who live in low and middle-income countries (3). The largest contributors to sepsis incidence and mortality among all age groups were diarrheal diseases and lower respiratory infections, respectively (2). It has been endemic for centuries since infections from different foci have accompanied mankind. In modern medicine, much has been studied and learned about sepsis, but therapeutic measures are still very limited, especially when a noxious hyperinflammatory state takes place. The COVID pandemic has increased public awareness of organ damage induced by sepsis, which is positive per se; on the other hand, it is surprising that laypeople and even health professionals are taken by so much surprise, as organ dysfunction has been recognized a result of various infections for decades. It is as if SARSCoV-2 is causing a previously unknown syndrome!

The Surviving Sepsis Campaign (SSC) began as a collaboration of members of the European Society of Intensive Care Medicine (ESICM), the International Sepsis Forum (ISF), and the Society of Critical Care Medicine (SCCM), with an aim at improving the diagnosis, survival, and management of septic patients (4). Its conception was at the ESICM meeting in Barcelona in 2002, and the first published guidelines date - 2004. It is a very important initiative, as sepsisassociated mortality is unacceptably high, septic patients are seen routinely by all practicing physicians, who may not be aware of all treatment options, whilst innovation and research are ongoing and updating on those is crucial (4).
Therefore, the aim of this editorial is to comment on the latest SSC guidelines, dated 2021 (5). We shall point out the main novelties proposed in them.

\section{Screening for sepsis}

Several screening tools for sepsis have been in use, the oldest but still much used one dating back to 1992, the systemic inflammatory response syndrome (SIRS) criteria (6). The SIRS criteria basically use vital signs (fever or hypothermia, heart rate, respiratory rate) plus the leukocyte count (a simple blood test), to establish the presence of inflammation; if infection is thought, or proved to be, the cause of inflammation, this is sepsis (6).

Recently, the quick Sequential Organ Failure Score (qSOFA) was proposed, based on the widely used Sequential Organ Failure Assessment (SOFA) criteria (1). This change had the purpose of defining sepsis as the presence of infection AND organ dysfunction. The advantages of this proposal are to draw attention to organ dysfunction, but its clear disadvantages are to screen patients when they already have organ failure, and not before.

The SSC 2021 (5) points out that, in the original derivation study, only $24 \%$ of infected patients had a qSOFA score of 2 or 3 , which stands out as evidence of the low sensitivity of the score as a screening tool, although the qSOFA was a good marker of severity. The panel, therefore, issued a strong recommendation against the use of qSOFA as a single screening tool. The Sepsis 3.0 paper was also criticized for not including the measurement of lactate levels in septic patients, since lactate elevation is often an early sign of hypoperfusion and microcirculatory dysfunction in sepsis. The panel recognizes that, although lactate alone is neither sensitive nor specific to establish the diagnosis of sepsis, it should be used as an adjunctive test, since the association of lactate level with mortality in infected patients is well recognized, and they therefore suggest lactate is measured in septic adult patients.

Address for Correspondence: Cristiane Lamas, Instituto Nacional de Cardiologia and Instituto Nacional de Infectologia Evandro Chagas, Fiocruz, Rio de Janeiro, Brazil Email: cristianelamas@gmail.com

Received: 14.02.2022 Accepted: 15.02.2022 Copyright (C2022 Heart, Vessels and Transplantation 


\section{Resuscitation in sepsis and septic shock}

The recommendations regarding resuscitation in septic patients with hypoperfusion or septic shock are still that they are managed with a $30 \mathrm{ml} / \mathrm{kg}$ in 3 hours infusion of crystalloids infusions. What is new it that capillary refill time is suggested as an adjunct measure to guide adequate resuscitation, when advanced hemodynamic monitoring is not available. This, as well as skin mottling and temperature of the extremities, have been validated as signs of tissue perfusion. Also balanced solutions, such as Ringer's lactate, are suggested instead of normal saline infusions, as the risk of hyperchloremic acidosis and acute kidney injury are less with the first and a metanalysis showed lower mortality when balanced crystalloids were used in sepsis (7). A suggestion against gelatins is also stated. Regarding the continuing administration of fluids in the first $\mathbf{2 4}$ hours, the panel issued a statement that there is insufficient evidence to advise on a restrictive vs. liberal strategy of fluid administration. Norepinephrine remains the vasopressor of first choice in patients in septic shock, but, very importantly, as this impacts on practice especially in lower resource settings, it is suggested that vasopressors may be initiated via peripheral veins, preferably antecubital veins, for up to 6 hours, so as not to delay the start of vasopressors. If there is an ongoing need for vasopressors in patients with septic shock, the panel suggests administering intravenous hydrocortisone.In patients with heart failure and a septic shock with signs of hypoperfusion despite adequate volume status and blood pressure, the panel suggests against the use of levosimendan. Levosimendan is a calciumsensitizing drug with inotropic and vasodilatory properties. A meta-analysis of seven randomized controlled trials comparing levosimendan with dobutamine showed that levosimendan was not superior to dobutamine in adults with sepsis in terms of mortality (8). The panel, therefore, suggests either adding dobutamine to norepinephrine or using epinephrine alone in this situation.

The goal of a mean arterial pressure (MAP) of 65 $\mathrm{mmHg}$ is recommended, and not higher levels of MAP. Targeting higher levels of MAP with vasopressors was associated with a higher risk of atrial fibrillation and did not improve survival in septic shock (9).

Other novelties not terribly relevant in clinical practice due to very limited availability are:
Angiotensin II has recently become available as a synthetic hormone for clinical use and it has marked vasoconstrictor effects. It has been studied in two clinical trials, but clinical experience in sepsis and demonstration of safety remains limited. Therefore, the panel considered that angiotensin should not be used as a first line agent but may have a role as an adjunctive vasopressor therapy.

Terlipressin is a prodrug, which is converted to lysine vasopressin by endothelial peptidases, producing a "slow release" effect resulting in a half-life of 6 hours. It has been studied in 9 clinical trials of patients with sepsis, but the SSC 2021 metanalysis showed no difference in mortality from sepsis compared to norepinephrine, and an increase in adverse events, especially digital and intestinal ischemia. This resulted in the panel issuing a weak recommendation against its use in patients with septic shock.

Selepressin is a highly selective V1 agonist, inducing vasoconstriction via stimulation of vascular smooth muscle. Compared to vasopressin, it does not have increased pro-coagulant activity, so much salt, and water retention, and nitric oxide, and corticosteroid release, and has been postulated as a potentially attractive non-catecholamine vasopressor alternative to norepinephrine. However, in two randomized trials in septic shock, it failed to demonstrate clinical superiority over norepinephrine, and it is not commercially available. Therefore, the panel issued a weak recommendation against its use as a first-line therapy.

\section{Admission to ICU}

Admission to ICU in up to 6 hours was suggested, as this improves outcomes (length of hospital stay, length of mechanical ventilation and mortality). However, when and where there are no ICU beds, appropriate treatment should not be delayed, irrespective of patient location.

\section{Antimicrobial administration}

Very importantly, regarding timing of antibiotic administration, guidelines have changed: in septic patients who are not in shock, the panel suggests a rapid assessment (clinical and laboratory) to rule in or out infection: if concern of infection persists, antimicrobials should be administered within $\mathbf{3}$ hours of presentation. For those who are thought highly likely of having infection or who are in septic shock, recommendation is to administer antimicrobials within 1 hour of presentation. 
The rationale behind this is: i) nearly a third of patients though to be septic have a non-infectious diagnosis and ii) mortality reduction associated with early antimicrobial therapy is highest in those with septic shock.

Regarding biomarkers to guide start of antibiotics, the panel suggest against using procalcitonin in this decision. This was based on the analysis of evidence from three randomized clinical trials that compared procalcitonin-guided protocols vs usual care for antibiotic initiation, which showed no difference in short-term mortality, length of hospitalization and length of ICU stay.
The SSC 2021 is more specific regarding empirical antibiotics when MRSA is considered a potential agent. It recommends antibiotics covering MRSA in those patients at high risk of this bacterium, and not using these when MRSA is not suspected. Table 1 shows situations with high risk for MRSA infection (10); it is important to notice that community-acquired MRSA and hospital-acquired MRSA often invade each other's "niche". The same logic applies to suspected fungal infections: if the patients are considered at high risk for fungal infections, antifungals are recommended, and if not high risk, antifungals are advised against. The document provides a table, which depicts situations of high risk for fungal infections.

\begin{tabular}{|l|l|l|}
\hline \multicolumn{3}{|l|}{ Table 1. Risk factors for MRSA infection in patients with sepsis or septic shock } \\
\hline $\begin{array}{l}\text { RISK FACTORS FOR } \\
\text { INFECTION WITH: }\end{array}$ & CA-MRSA & h-MRSA \\
\hline & children & institutionalized people \\
\hline & athletes & individuals with cystic fibrosis \\
\hline & military personnel & patients frequently admitted to hospital \\
\hline+++++++++++++ & intravenous drug users & \\
\hline & $\begin{array}{l}\text { people who live in underserved areas, } \\
\text { in crowded conditions }\end{array}$ \\
\hline & HIV positive individuals & \\
\hline CA-MRSA=community-acquired methicillin resistant Staphylococcus aureus; h=MRSA= hospital-acquired MRSA \\
\hline
\end{tabular}

\section{Ventilation strategies}

Regarding ventilation, two new strategies are recommended: i) septic patients with hypoxemic respiratory failure, high flow nasal oxygen is suggested over non-invasive ventilation, and for those with sepsis related ARDS, veno-venous extracorporeal membrane oxygenation (ECMO) is suggested when mechanical ventilation fails, provided ECMO is offered by experienced centers.

\section{Additional therapies}

For adults with septic shock, severe metabolic acidemia $(\mathrm{pH} \leq 7.2)$ and acute kidney injury (AKIN score of 2 or 3 ), the panel suggested using sodium bicarbonate therapy. For adults with sepsis or septic shock, the panel suggested against using IV - intravenous vitamin C.

\section{Goals of care and follow up}

The panel recommended, as best practice, discussing goals of care and prognosis with adult patients and their families early (within 72 h) over late. Also integrating principles of palliative care is recommended, when appropriate, to address patient and family suffering. However, it suggested against routine formal palliative care consultation for all patients over palliative care consultation based on clinicians' judgment.

Regarding discharge from ICU, the panel recommended: i) using a critical care transition program, upon transfer to the floor, ii) reconciling medications at both ICU and hospital discharge, iii) a hospital discharge summary with complete information about the ICU stay, sepsis and related diagnoses, treatments, and common impairments after sepsis, iv) screening for economic and social support (including housing, nutritional, financial, and spiritual support), and making referrals where available to meet these needs, and v) for those with new impairments post sepsis, follow-up with clinicians able to support and manage new and long-term sequelae is recommended. 
In summary, Surviving Sepsis Campaign guidelines are important to all clinicians, whichever is the level of care they practice in. They provide the best level of evidence available at the time of their publication and should be carefully read and appreciated.

Cristiane Lamas Instituto Nacional de Cardiologia and Instituto Nacional de Infectologia Evandro Chagas, Fiocruz, Rio de Janeiro, Brazil

Peer-review: Internal Conflict of interest: None to declare Authorship: C.L. Acknowledgement and funding: None to declare

\section{References}

1.Singer M, Deutschman CS, Seymour CW, Shankar-Hari $M$, Annane J, Bauer $M$, et al. The third international consensus definitions for sepsis and septic shock (Sepsis-3). JAMA 2016; 315: 801-10. doi: 10.1001/jama.2016.0287.

2.Rudd KE, Johnson SC, Agesa KM, Shackelford KA, Tsoi $D$, Kievlan DR, et al. Global, regional, and national sepsis incidence and mortality, 1990-2017: analysis for the Global Burden of Disease Study. Lancet 2020; 395: 20011. doi: 10.1016/S0140-6736(19)32989-7

3.Global report on the epidemiology and burden of sepsis: current evidence, identifying gaps and future directions. Geneva: World Health Organization; 2020. Licence: CC BY-NC-SA 3.0 IGO.

4. History. Surviving Sepsis Campaign, Available at: URL: https://www.sccm.org/SurvivingSepsisCampaign/About -SSC/History

5.Evans L, Rhodes A, Alhazzani W, Antonelli M, Coopersmith CM, French $C$, et al. Surviving Sepsis Campaign: International Guidelines for Management of Sepsis and Septic Shock 2021. Crit Care Med. 2021; 49: e1063-e1143. doi: 10.1097/CCM.0000000000005337 6.Bone RC, Balk RA, Cerra FB, Dellinger RP, Fein AM, Knaus WA, et al. Definitions for sepsis and organ failure and guidelines for the use of innovative therapies in sepsis. The ACCP/SCCM Consensus Conference Committee. American College of Chest Physicians/Society of Critical Care Medicine. Chest 1992; 101: 1644-55. doi: 10.1378/chest.101.6.1644

7.Rochwerg B, Alhazzani W, Sindi A, Heels-Ansdell D, Thabane L, Fox-Robichaud $E$, et al. Fluid resuscitation in sepsis: a systematic review and network meta-analysis. Ann Intern Med 2014; 161: 347-55.

8.Bhattacharjee S, Soni KD, Maitra S, Baidya DK. Levosimendan does not provide mortality benefit over dobutamine in adult patients with septic shock: a metaanalysis of randomized controlled trials. J Clin Anesth 2017; 39:67-72.

9.Hylands $\mathrm{M}$, Moller MH, Asfar P, Toma A, Frenette AJ, Beaudoin $\mathrm{N}$, et al. A systematic review of vasopressor blood pressure targets in critically ill adults with hypotension. Can J Anaesth 2017; 64: 703-15.

10.Turner NA, Sharma-Kuinkel BK, Maskarinec SA, Eichenberger EM, Shah PP, Carugati $M$, et al. Methicillin-resistant Staphylococcus aureus: an overview of basic and clinical research. Nat Rev Microbiol 2019; 17: 203-18. doi: 10.1038/s41579-0180147-4. 